\title{
Updated Results on the UHECR Hotspot Observed by the Telescope Array Experiment
}

\author{
K. Kawata ${ }^{* a}$, A. di Matteo ${ }^{b}$, T. Fujiic ${ }^{c}$, D. Ikeda ${ }^{d}$, D. Ivanov ${ }^{e}$, C. C. H. Jui ${ }^{e}$, E. Kido ${ }^{a}$, \\ J. P. Lundquist ${ }^{e}$, J. N. Matthews ${ }^{e}$, S. Nagataki ${ }^{f}$, T. Nonaka ${ }^{a}$, S. Ogio ${ }^{g}$, T. Okuda ${ }^{h}$, \\ G. Rubtsov ${ }^{i}$, H. Sagawa ${ }^{a}$, T. Sako ${ }^{a}$, N. Sakurai ${ }^{g}$, M. Takeda $^{a}$, R. Takeishi ${ }^{j}$, A. Taketa ${ }^{d}$, \\ G. B. Thomson ${ }^{e}$, P. Tinyakov ${ }^{b, i}$, I. Tkachev ${ }^{i}$, S. Troitsky ${ }^{i}$ for the Telescope Array \\ Collaboration. \\ ${ }^{a}$ Institute for Cosmic Ray Research, University of Tokyo, Kashiwa, Chiba, Japan \\ ${ }^{b}$ Service de Physique Théorique, Université Libre de Bruxelles, Brussels, Belgium \\ ${ }^{c}$ The Hakubi Center for Advanced Research and Graduate School of Science, Kyoto University, \\ Sakyo-ku, Kyoto, Japan \\ ${ }^{d}$ Earthquake Research Institute, University of Tokyo, Bunkyo-ku, Tokyo, Japan \\ ${ }^{e}$ High Energy Astrophysics Institute and Department of Physics and Astronomy, University of \\ Utah, Salt Lake City, Utah, USA \\ ${ }^{f}$ Astrophysical Big Bang Laboratory, RIKEN, Wako, Saitama, Japan \\ ${ }^{g}$ Graduate School of Science, Osaka City University, Osaka, Osaka, Japan \\ ${ }^{h}$ Department of Physical Sciences, Ritsumeikan University, Kusatsu, Shiga, Japan \\ ${ }^{i}$ Institute for Nuclear Research of the Russian Academy of Sciences, Moscow, Russia \\ ${ }^{j}$ Department of Physics, Sungkyunkwan University, Jang-an-gu, Suwon, Korea \\ E-mail: Eawatadicrr.u-tokyo.ac.jp
}

The Telescope Array Experiment has observed an indication of intermediate-scale anisotropy in the UHECR arrival directions, called the Hotspot, with $E>57 \mathrm{EeV}$ around the Ursa Major using the first 5-year data during a period between May 2008 and May 2013 collected by the TA surface detector array. The chance probability of this hotspot in an isotropic cosmic-ray sky was calculated to be $3.4 \sigma$ (post trial). In this paper, we will report on an update of this result using the 11-year data collected by the TA surface detectors with more than doubled exposure since the first publication.

36th International Cosmic Ray Conference -ICRC2019-

July 24th - August 1st, 2019

Madison, WI, U.S.A.

* Speaker. 


\section{Introduction}

The Telescope Array (TA) and the Pierre Auger Observatory (Auger) are the largest UHECR detectors in north and south hemispheres, respectively. They have both obtained some hints/evidence of anisotropy in the UHECR directions. Recent anisotropy measurements and the efforts to identify UHECR sources are summarized in Ref. [U]. The Auger group has claimed that the starburst model fits the observed UHECR distribution better than the hypothesis of isotropy with a statistical significance of $4.0 \sigma[\nabla]$. They also has have claimed a dipole-like anisotropy with an amplitude of $6.5 \%$ for cosmic rays with energy greater than $8 \mathrm{EeV}$. The anisotropy has a statistical significance of $5 \sigma$ [3] .

The TA collaboration has first reported a hotspot of UHECRs, with $E>57 \mathrm{EeV}$, in the first 5-years observation period from 2008 May to 2013 May [四]. The "hotspot" is located near the direction of the Ursa Major cluster, and extends to $\sim 20^{\circ}$ angular scale. The chance probability of detecting this hotspot in an isotropic cosmic-ray sky was calculated to be $3.7 \times 10^{-4}(3.4 \sigma)$ assuming $15^{\circ}, 20^{\circ}, 25^{\circ}, 30^{\circ}$, and $35^{\circ}$ oversampling radius circles were searched. After the report of hotspot, the TA group found that the flux has a deficit in the energy range $10^{19.2}<E<10^{19.75} \mathrm{eV}$ and an excess in $E>10^{19.75} \mathrm{eV}(\sim 57 \mathrm{EeV})$, respectively, compared with the average flux of the other sky using the 7-year data [5]. The post-trial probability of this spectral anisotropy appearing by chance from the isotropic distribution is estimated to be $9 \times 10^{-5}$, which corresponds to $3.74 \sigma$. Recent anisotropy studies by the TA are summarized in Ref. [焑].

In this paper, we present the updated results on the TA hotspot with the latest 11-year data collected by the Telescope Array surface detector array.

\section{Telescope Array Experiment}

The Telescope Array (TA) is the largest cosmic-ray detector in the northern hemisphere. It consists of a surface detector (SD) array [ $[\mathbf{D}]$ and three fluorescence detector (FD) stations [ [ 8 , Q $]$ ]. The TA has been fully operational in Millard County, Utah, USA $\left(39.30^{\circ} \mathrm{N}, 112.91^{\circ} \mathrm{W}\right.$; about $1,400 \mathrm{~m}$ above sea level), since 2008. The TA SD array consists of 507 plastic scintillation detectors of $3 \mathrm{~m}^{2}$ area located on a square grid with $1.2 \mathrm{~km}$ separation and makes measurements of the footprints of extensive air showers when they arrive at the Earth's surface. The SD array has an area of approximately $700 \mathrm{~km}^{2}$. For more details, see Ref. [ए0]. The TA SD array observes cosmic rays from $\sim 1 \mathrm{EeV}$ to $100 \mathrm{EeV}$ using the extensive-air-shower technique of a scintillation detector array with a duty cycle near $100 \%$ regardless of weather conditions, and with a wide field of view. These capabilities give us an unbiased survey of the northern sky. The telescope stations observe the sky over the SD array and make measurements of the longitudinal development of the air showers as they traverse the atmosphere.

\section{Results and Discussions}

The updated results on the TA hotspot were reported at the previous conferences using the 7-year [U]] and 9-year data [12], respectively. In this work we test this result using the 11-year data collected by the TA SD array. We have observed 168 events with $E>57 \mathrm{EeV}$ in 11 years, which is 

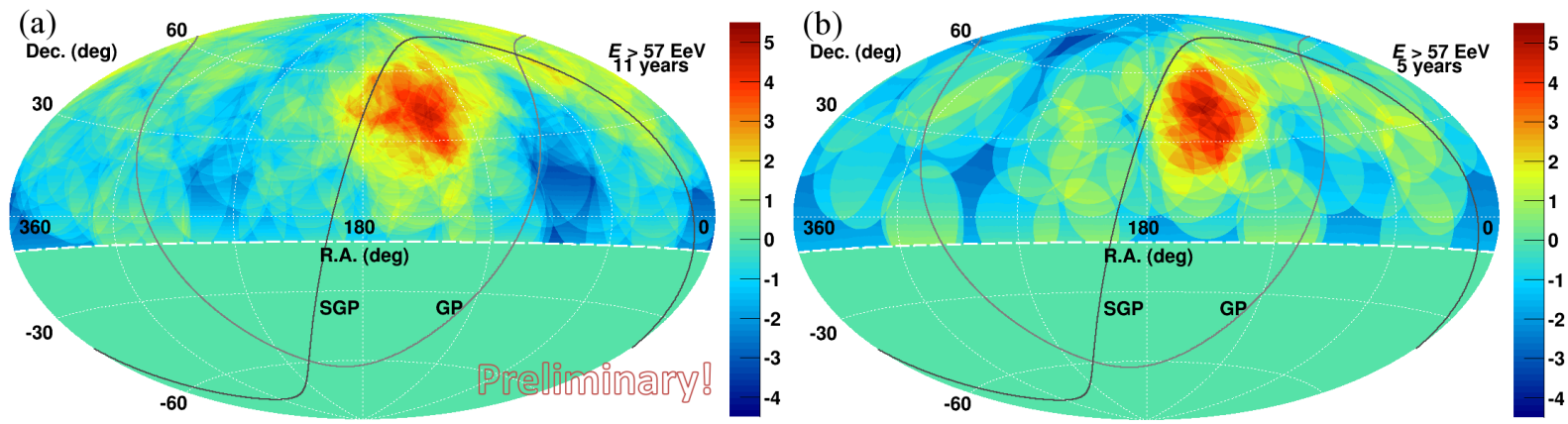

Figure 1: (a) A significance map of the UHECR events with $E>57 \mathrm{EeV}$ for 11 years of TA data (May 2008 - May 2019) in the equatorial coordinates. Events are smoothed by $25^{\circ}$ oversampling radius circle, which is defined in this paper. (b) A significance map of the UHECR events with $E>57 \mathrm{EeV}$ for events observed in the 1 st 5 years of TA data (May 2008 - May 2013). Events are smoothed by $20^{\circ}$ oversampling radius circle according to our original paper [四]. The solid curves indicate supergalactic plane (SGP) and the galactic plane (GP).

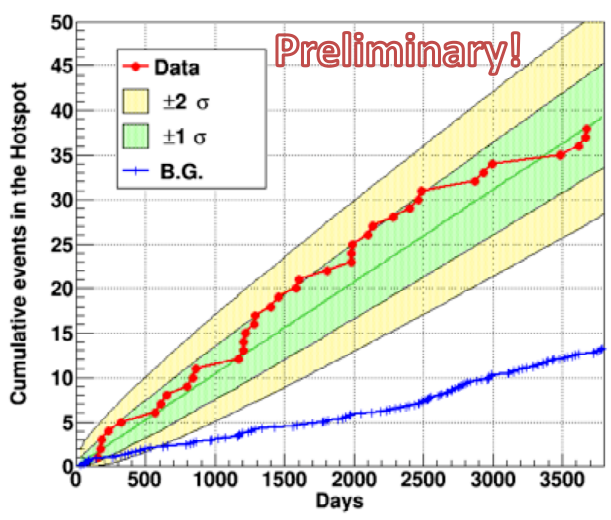

Figure 2: Number of cumulative events of the hotspot region (Red curve), and cumulative background events (Blue curve), respectively, above $57 \mathrm{EeV}$. The green and yellow shaded areas show $\pm 1 \sigma$ and $\pm 2 \sigma$ deviations from the rate of data observation respectively, assuming a linear increase in rate.

approximately double statistics of the first 5-year observation. These events are summed over different five oversampling radius circles, $15^{\circ}, 20^{\circ}, 25^{\circ}, 30^{\circ}$, and $35^{\circ}$. The centers of tested directions are on a $0.1^{\circ} \times 0.1^{\circ}$ grid in the equatorial coordinates. We then search for the maximum significance over all grid points and five oversampling radius circles. We found the maximum significance of $5.1 \sigma$ at a position R.A. $=144.3^{\circ}$, and Dec. $=40.3^{\circ}$ with $25^{\circ}$ oversampling radius circle. The chance probability of the 11-year hotspot in an isotropic sky is estimated to be $2.1 \times 10^{-3}(2.9 \sigma)$. Figure $\square$ (a) shows the significance maps of the UHECR events with $E>57 \mathrm{EeV}$ for 11 years with $25^{\circ}$ radius circle, compared with our previous result for the 1 st 5 years of data with $20^{\circ}$ shown in Fig.W (b) [四]. The 11-year hotspot looks larger size than the 5-year hotspot (the number of background events in $25^{\circ}$ radius circle is $50 \%$ higher than that of $20^{\circ}$ radius circle). It has extended all the way to the supergalactic plane (SGP), and is irregular in shape. Therefore a circular oversampling shape is not really appropriate. In that case, the significance of such an excess might be underestimated. 
In Fig.1 (a), a marginal excess is seen along the SGP (around the Perseus-Pisces Supercluster) at the local significance of $\sim 3 \sigma$.

We divided the 11-year data into the first 5 years and the second 6 years. The significance at the hotspot position is $5.0 \sigma$ for the first 5 years and $2.2 \sigma$ for the second 6 years, respectively, with $25^{\circ}$ radius circle. Figure $\square$ shows the cumulative events inside the hotspot circle defined by the 11-year dataset to check a deviation from the linear increase. The green and yellow bands in this figure represent the $\pm 1 \sigma$ and $\pm 2 \sigma$ allowed regions, respectively. The increase rate of the events inside the hotspot circle is consistent with a constant within $\pm 1 \sigma$ fluctuation.

\section{Summary}

In this paper, the TA hotspot analysis was updated using the 11-year observation. We found the local significance of $5.1 \sigma$ at a position R.A. $=144.3^{\circ}$, and Dec. $=40.3^{\circ}$ with $25^{\circ}$ oversampling radius circle. The chance probability of the 11-year hotspot in an isotropic sky is estimated to be $2.1 \times 10^{-3}(2.9 \sigma)$. The TA will continuously observe to verify our current results. In addition, the TA $\times 4$ experiment has partly started its data taking in 2019 [[13], which will extend the size of the TA SD by a factor of 4 in near future to collect data at a faster rate.

\section{Acknowledgments}

The Telescope Array experiment is supported by the Japan Society for the Promotion of Science(JSPS) through Grants-in-Aid for Priority Area 431, for Specially Promoted Research JP21000002, for Scientific Research (S) JP19104006, for Specially Promote Research JP15H05693, for Scientific Research (S) JP15H05741 and for Young Scientists (A) JPH26707011; by the joint research program of the Institute for Cosmic Ray Research (ICRR), The University of Tokyo; by the U.S. National Science Foundation awards PHY-0601915, PHY-1404495, PHY-1404502, and PHY-1607727; by the National Research Foundation of Korea (2015R1A2A1A01006870, 2015R1A2A1A15055344, 2016R1A5A1013277, 2016R1A2B4014967, 2017R1A2A1A05071429); by the Russian Academy of Sciences, RFBR grant 16-02-00962a (INR), IISN project No. 4.4502.13, and Belgian Science Policy under IUAP VII/37 (ULB). The foundations of Dr. Ezekiel R. and Edna Wattis Dumke, Willard L. Eccles, and George S. and Dolores Dore Eccles all helped with generous donations. The State of Utah supported the project through its Economic Development Board, and the University of Utah through the Office of the Vice President for Research. The experimental site became available through the cooperation of the Utah School and Institutional Trust Lands Administration (SITLA), U.S. Bureau of Land Management (BLM), and the U.S. Air Force. We appreciate the assistance of the State of Utah and Fillmore offices of the BLM in crafting the Plan of Development for the site. Patrick Shea assisted the collaboration with valuable advice on a variety of topics. The people and the officials of Millard County, Utah have been a source of steadfast and warm support for our work which we greatly appreciate. We are indebted to the Millard County Road Department for their efforts to maintain and clear the roads which get us to our sites. We gratefully acknowledge the contribution from the technical staffs of our home institutions. An allocation of computer time from the Center for High Performance Computing at the University of Utah is gratefully acknowledged. 


\section{References}

[1] O. Deligny, K. Kawata and P. Tinyakov, PTEP., 12A104 (2017)

[2] A. Aab et al. (The Pierre Auger Collaboration), ApJL, 853, L29 (2018)

[3] A. Aab et al. (The Pierre Auger Collaboration), Science, 357, 1266 (2017)

[4] R. U. Abbasi et al. (The Telescope Array Collaboration), ApJL, 790, L21 (2014)

[5] R. U. Abbasi et al. (The Telescope Array Collaboration), ApJ 862, 91 (2018)

[6] K. Kawata et al. (The Telescope Array Collaboration), EPJ Web Conf. (proceedings of UHECR 2018), 210, 01004 (2019)

[7] H. Kawai et al.(The Telescope Array Collaboration), Nucl. Phys. B Proc. Suppl., 175, 221 (2008)

[8] H. Tokuno, et al. (The Telescope Array Collaboration), NIM-A, 676, 54 (2012)

[9] J. N. Matthews et al. (The Telescope Array Collaboration), 30th ICRC (Merida), 5, 1157 (2007)

[10] T. Abu-Zayyad et al. (The Telescope Array Collaboration), NIM-A, 689, 87 (2012)

[11] K. Kawata et al. (The Telescope Array Collaboration), Proc. of the 34th ICRC (Den Haag), PoS(ICRC2015)276 (2015)

[12] S. Troitsky et al. (The Telescope Array Collaboration), Proc. of the 35th ICRC (Busan), PoS(ICRC2017)548 (2017)

[13] E. Kido et al. (The Telescope Array Collaboration), EPJ Web Conf. (proceedings of UHECR 2018), 210, 06001 (2019) 